Short Report

\title{
T Cell Therapy for Nasopharyngeal Carcinoma
}

\section{S Basso, M Zecca, P Merli, A Gurrado, S Secondino, G Quartuccio, I Guido, P Guerini, G Ottonello, N Zavras, R Maccario, P Pedrazzoli, P Comoli $\bowtie$}

Pediatric Hematology/Oncology, Research Laboratories, and Medical Oncology, Fondazione IRCCS Policlinico S. Matteo, Pavia, Italy.

$\triangle$ Corresponding author: Dr. Patrizia Comoli, M.D., Pediatric Hematology/Oncology, Fondazione IRCCS Policlinico S. Matteo, V.le Golgi 19, 27100 Pavia, Italy. Tel: (39)-0382-502716; Fax: (39)-0382-527976; e-mail: pcomoli@smatteo.pv.it.

(c) Ivyspring International Publisher. This is an open-access article distributed under the terms of the Creative Commons License (http://creativecommons.org/ licenses/by-nc-nd/3.0/). Reproduction is permitted for personal, noncommercial use, provided that the article is in whole, unmodified, and properly cited.

Received: 2011.04.30; Accepted: 2011.06.02; Published: 2011.06.03

\begin{abstract}
Among the novel biologic therapeutics that will increase our ability to cure human cancer in the years to come, T cell therapy is one of the most promising approaches. However, with the possible exception of tumor-infiltrating lymphocytes therapy for melanoma, clinical trials of adoptive T-cell therapy for solid tumors have so far provided only clear proofs-of-principle to build on with further development. Epstein-Barr virus (EBV)-associated malignancies offer a unique model to develop $\mathrm{T}$ cell-based immune therapies, targeting viral antigens expressed on tumor cells. In the last two decades, EBV-specific cytotoxic T-lymphocytes (CTL) have been successfully employed for the prophylaxis and treatment of EBV-related lymphoproliferative disorders in immunocompromised hosts. More recently, this therapeutic approach has been applied to the setting of EBV-related solid tumors, such as nasopharyngeal carcinoma. The results are encouraging, although further improvements to the clinical protocols are clearly necessary to increase anti-tumor activity. Promising implementations are underway, including harnessing the therapeutic potential of CTLs specific for subdominant EBV latent cycle epitopes, and delineating strategies aimed at targeting immune evasion mechanisms exerted by tumor cells.
\end{abstract}

Key words: nasopharyngeal carcinoma, T-cell therapy, cytotoxic T lymphocytes, Epstein-Barr virus.

\section{Introduction}

The development of new agents targeting important cellular pathways involved in cancer progression, although promising, has so far resulted in relatively short-term benefits for the majority of patients with advanced malignancy. Cell therapy can be numbered among the novel biologic therapeutics that will increase our ability to cure human disease in the years to come. Despite the great potential, $\mathrm{T}$ cell therapy for cancer still has a marginal role in the management of patients with neoplasia. This is due to limitations inherent to the technologies and products employed, and, more importantly, to the financial and structural requirements that are associated with cell therapy [1-3].

Clinical application was first attempted by Rosenberg and colleagues in 1985 by using LAK cells, infused with IL-2 into patients with different advanced malignancies [4]: response was observed in four kinds of solid tumors, thus paving the way to further investigations. Afterwards, several experimental and clinical studies were conducted: cell types tested included CIK cells [5], tumor infiltrating lym- 
phocytes (TILs) $[6,7,8]$ and other T-cells variously manipulated [1,3,9-11], which were used in different settings, from solid cancers to the transplantation field.

Among the factors that so far limited a wider use of $\mathrm{T}$ cell therapy for human tumors may be the very low frequency of tumor-specific lymphocytes circulating in patients with cancer, or the limited ability to induce $\mathrm{T}$ cell lines with protective antitumor activity with current knowledge and available technology. With the exception of TIL therapy in melanoma, the only other human solid cancer setting in which tumor-specific $T$ cells have been employed with success is virus-related tumors. Indeed, when the frequency of circulating $\mathrm{T}$ cells against the target antigen on a tumour is high, as is the case for viral antigens, $\mathrm{T}$ cell therapy can be very effective in destroying large tumours in humans. In this context, EBV-positive malignancies provide an optimal model system to test and ameliorate cellular therapies: the first very encouraging results were reached with prophylaxis and treatment of posttransplant lymphoproliferative disease [9-11]; the success of this approach has fostered research in other, more complex areas, such as EBV-related solid tumors.

\section{Nasopharyngeal carcinoma}

Nasopharyngeal carcinoma (NPC) is a rare cancer in the Western hemisphere where its incidence is approximately 1 case per 100,000 individuals; conversely there are some areas, such as Southern China, Southeast Asia, the Mediterranean basin and Alaska, where it reaches 80 per 100,000 individuals: moreover in the western hemisphere the tumor histology differs from the endemic form [12]. All these differences suggest that an important role in the pathogenesis is played by genetic and environmental factors.

The current WHO classification defines nasopharyngeal cancer a carcinoma that shows light microscopic or ultrastructural evidence of squamous differentiation. It encompasses squamous cell carcinoma, associated with behavioural risk factors such as alcohol and tobacco use, non keratinizing carcinoma (differentiated and undifferentiated) and basaloid squamous cell carcinoma [13]. The most common pediatric nasopharyngeal carcinoma is the non-keratinizing undifferentiated carcinoma [14] and is associated with EBV in practically $100 \%$ of cases [13].

Although NPC is serologically and biologically associated with Epstein-Barr Virus, NPC cells express only a limited pattern of EBV genes (the so-called latency II pattern) which comprises non-coding RNAs (EBERs, BARTs), the nuclear antigen EBNA1 and surface antigens LMP1 and LMP2 [15], immunogens that are weak, albeit capable of inducing a T-lymphocyte response .

In most cases the tumor presents as a painless mass in the upper neck, with possible cervical lymphadenopathy. The most common pattern of tumor diffusion is local infiltration which, given the limitrophe structures, may cause serous otitis, hearing difficulties, nasal obstruction, epistaxis, dysphasia, dysphonia and dysphagia. NPC can metastatize to lung, bone, mediastinum, bone marrow and visceral organs $[13,16,17]$. Paraneoplastic syndromes may also be present, in most cases related to tumor dissemination or relapse, such as hypertrophic osteoarthropathy, leukemoid reaction, FUO, dermatomyositis, and inappropriate $\mathrm{ADH}$ secretion syndrome [18].

The extent of the tumor at diagnosis is described by the TNM classification of the American Joint Committee on Cancer; in children, as in adults, the TNM stage at the time of diagnosis correlates with outcome [19]. However, with more advanced and improved treatments, while the presence of metastases continues to be associated with poor outcome, $\mathrm{T}$ and $\mathrm{N}$ staging are losing prognostic significance [20, 21].

NPC is highly sensitive to both radio- and chemotherapy. Radiotherapy is a cornerstone of first line therapy and it is the standard treatment for early-stage NPC (T1-2a; N0; M0): patients with local disease can be adequately treated with radiation alone, with a 5-years control ranging from 80 to $95 \%$ of cases [22-25] .

Treatment strategies for locally advanced NPC consists mainly of platinum based chemotherapy in conjunction with radiotherapy, and yields an overall response rate of about $90 \%$, with complete response ranging from 20 to $50 \%$ [26-28]. Induction treatment is able to improve local regional control, which translates into long-term specific survival benefits. In local-regional recurrent nasopharyngeal cancer not amenable for reirradiation, combination cisplatin-based chemotherapy is a standard first line treatment, with response rates of $40-80 \%$, mainly depending on the site of lesions, previous treatment, disease free interval $[28,29]$. No standard second-line chemotherapy has been defined, but a recent study reported an $11 \%$ response rate in recurrent and metastatic disease by combining target therapy (cetuximab) to carboplatin [30]. However, the benefits of this treatment are generally short-lived. Second line therapies in refractory/relapsing patients usually have little effect on the natural history of the disease, and there is therefore a need to develop additional forms of 
treatment, particularly ones that lack overlapping toxicities with radiochemotherapy [31,32].

\section{Cell therapy for NPC}

\section{Clinical results}

In the last 15 years, a number of reports demonstrated the effectiveness of ATCT directed against EBV antigens for the treatment of EBV-related hematological malignancies in the immunocompromised host [3,9-11,33]. EBV-related posttransplant lymphoproliferative disease (PTLD) constitutes a highly immunogenic lymphoproliferation whose onset is greatly favoured by the host immunodeficiency status. Thus, T cell therapy in this setting is expected to have a great chance of success. Adoptive transfer of polyclonal CTLs specific for viral latency antigens in the context of EBV-associated malignancies arising in the immunocompetent host, such as nasopharyngeal carcinoma (NPC), is hampered by a number of factors [34]. EBV-specific CTLs are dominated by reactivity against viral proteins not expressed by these tumors. Moreover, the transferred CTLs have to compete with endogenous lymphocytes for cytokines and biological niches, and, once CTLs reach the tumor site, they have to overcome the inhibitory barriers exerted by the tumor environment. Notwithstanding these limitations, the results of the clinical cell therapy trials conducted so far in NPC patients demonstrate that administration of an avid anti-tumour $\mathrm{T}$ cell targeting a highly expressed antigen can result in cancer regression [35,36].

To date, a total of 57 patients with NPC were treated within phase I-II clinical trials [35-41]. In a phase I study, Comoli et al. [35] enrolled 10 patients with stage IV NPC in progression after conventional radiotherapy and chemotherapy. Patients underwent multiple (from 2 to 23) infusions of autologous EBV-specific CTLs with a dose ranging from 2 to $8 \times 10^{7}$ cells $/ \mathrm{m}^{2}$, accompanied by administration of low-dose $\left(1 \times 10^{6} \mathrm{U}\right)$ recombinant IL-2 with the purpose of prolonging T-lymphocyte life-span. There were no major adverse events, although two patients suffered from moderate inflammatory reactions at the tumor site. Control of disease progression was obtained in six of ten patients (two with partial response and four with stable disease). More importantly, it was demonstrated that tumor control related to cell therapy did occur, and was associated to the emergence or increase in LMP2-specific responses in the peripheral blood. In the same year, Straathof and colleagues [36] published data from 10 patients with advanced NPC. In a recent update [37] Louis added 13 new patients to the previous 10 (whose follow-up was extended), for a total of 23 patients treated. Of these patients (all but one with stage III-IV NPC) eight were in disease remission at the time of the first infusion and 15 had active disease. They were treated with a mean of 2 infusions with a dose ranging from $2 \times 10^{7}$ cells $/ \mathrm{m}^{2}$ to $2 \times 10^{8}$ cells $/ \mathrm{m}^{2}$, in the absence of IL-2 use. The therapy was well tolerated: only one patient experienced marked swelling at the tumor site requiring a tracheostomy. Of the eight patients treated in remission five remained disease-free for 25 to 82 months and three suffered from disease relapse. Of the other 15 patients treated with active disease ten showed control of the disease ( 5 with Complete Response, 2 with Partial Response, 3 with Stable Disease), while the other five progressed. Prior to these two studies, one pilot study in China showed that the adoptive transfer of autologous EBV-targeted CTLs induced antiviral responses but no clinical responses in 4 NPC patients [40], while adoptive transfer of allogeneic EBV-specific CTL induced a clinical response in a patient with advanced-stage NPC, likely associated to a boost in endogenous LMP2-specific response [41].

The data obtained with these early studies prompted further efforts aimed at enhancing the expansion potential of infused T-cells, by means of a lymphodepleting treatment prior to CTL infusion, as demonstrated in the context of T-cell therapy for melanoma [42,43].

In a pilot study, 8 patients with loco-regional or metastatic refractory/recurrent NPC were given anti-CD45 monoclonal antibody treatment, followed by escalating doses of polyclonal EBV-specific CTL. After transitory lymphodepletion, and increase in the circulating levels of IL-15, 3 objective responses were seen, in the patients who showed higher increase in their peripheral blood frequency of EBV-specific $T$ cells after CTL infusion [38]. Secondino and colleagues [39] enrolled 11 heavily pre-treated patients with active stage IV NPC, who received non-myeloablative lymphodepleting chemotherapy consisting of cyclophosphamide and fludarabine: two doses of autologous EBV-specific CTLs (median dose of $3.7 \times 10^{8}$ cells) were subsequently infused, two weeks apart, supported by low-dose IL-2. Control of disease was obtained in six of eleven patients (3 with stable disease, 2 with partial response and one with minor response). Two patients experienced mild to moderate swelling at the disease site. Notwithstanding the encouraging preliminary results, the use of lymphodepleting preparative regimens as a mean to overcome the inhibitory checkpoints devised by the tumor cells is a strategy that needs further optimization. 
To summarize, data collected from a total of 57 patients receiving polyclonal, EBV-polyspecific CTL were as follows:

1) of 30 patients with advanced, active NPC, treated with autologous or allogeneic, polyclonal EBV-specific CTL infusions, 17 (56\%) achieved control of the disease (defined as either tumor regression or disease stabilization)

2) of 19 patients with advanced, active NPC, treated with autologous, polyclonal EBV-specific CTL infusions preceded by a lymphodepleting regimen 9 $(47 \%)$ achieved control of the disease

3) of 8 patients treated in disease remission, although at great risk of relapse, at the time of the first infusion, 5 (62\%) remained disease-free for 25 to 82 months and 3 suffered from relapsing disease

\section{Considerations on clinical data and on future strategies to ameliorate outcome}

$\mathrm{T}$ cell therapy, although now frequently used in the hematopoietic transplantation setting, has not yet been widely introduced in the clinical oncologic field and is currently limited to research protocols in highly-specialized centres. For solid tumors, the majority of the literature available nowadays refers to treatment of melanoma [42]. The phase I-II studies of EBV-targeted T cell therapy for NPC, taken together, provide one of the larger series for non-melanoma solid malignancies. These studies provide new perspectives on the optimal treatment for metastatic or recurrent disease; indeed, to date, cisplatin-based chemotherapy is considered the preferred regimen for such patients: response rates are above $50 \%$ and median survival is approximately 12 months [26]. However, the choice for second-line regimens in patients with unsatisfactory responses to a platinum-based treatment remains unclear [32].

In the cell therapy trials described, the patients enrolled were a heavily pre-treated cohort with a very poor prognosis. In these patients, further conventional treatment would likely lead to a very high risk of toxicity, ranging from $26 \%$ after re-irradiation with high dose radiotherapy [44] to $89 \%$ after conventional cytotoxic protocols [45]. In the cohort treated with CTLs, there were only five out of 52 patients (less than $10 \%$ ) who suffered from swelling at the tumor site; of these, four had mild to moderate inflammatory reactions $[35,39]$ and only one had a major complication [36]. In particular this patient, who presented bulky disease and pre-existing facial swelling, two days after the first infusion, developed marked worsening of the tumefaction requiring a tracheostomy: the authors performed a needle biopsy which showed tumor cells, but not inflammatory cells, suggesting tumor pro- gression rather than an inflammatory reaction. Thus the safety and the feasibility of this approach, even at the highest doses reached, is more than a supposition.

The second point to take into consideration is the efficacy of this therapy: taken grossly together all these data show a response rate ranging from 47 to $64 \%$, thus perfectly in line with other types of second-, third- or more advanced therapy [32]. For the patients treated in remission, given the small size of the cohort, the role of infused CTLs remains unclear.

But, more importantly, these data raise a number of questions, which remains, to date, unresolved. First of all, what is the role and the best timing of adoptive T-cell therapy in NPC? The studies published to date indicate that CTLs could possibly be administered earlier the course of treatment and in combination with conventional therapies (for example as consolidation treatment after achieving response to second line therapy) [39].

Furthermore, immune escape can occur even in instances when polyclonal antigen-specific $\mathrm{T}$ cells are infused [46]: Gottschalk et al reported a patient with EBV-positive lymphoproliferative disease, who failed EBV-specific T-cell therapy, in which the tumor virus had deleted immunodominant EBV epitopes [47]. In the setting of NPC immune escape could represent an even more relevant problem. A strategy to overcome this phenomenon, and to increase clinical efficacy through better targeting, could be to ameliorate the quality of the cell product. In this perspective, efforts are being made towards augmenting the pool of T-cells specific for the subdominant antigens expressed on EBV latency II tumor cells within the infused product, with the aim of increasing $\mathrm{T}$ cell therapy efficacy. In detail, the subdominant component of the EBV-specific immune response directed towards latent membrane proteins LMP1 and LMP2 has been shown to expand, by stimulation with dendritic cells or EBV-LCL genetically modified to express the antigens [48-50]. In a pilot study enrolling EBV-positive Hodgkin or non-Hodgkin lymphoma, 5 of 6 patients with active relapsed disease showed a tumor response after infusion of autologous LMP2-specific CTL [51]. Similar studies in NPC cohorts are warranted.

A barrier to the function of infused EBV-specific CTLs in immunocompetent hosts is the display of tumor-mediated immune evasion strategies [52]. To improve the resistance of CTLs to tumor-derived inhibitory cytokines, Bollard et al. have shown that EBV-specific CTL made transgenic for a dominant-negative TGF- $\beta$ receptor, in which the intracellular signaling domain is truncated, are rendered resistant to the devastating effects of TGF- $\beta$, secreted by Hodgkin tumor cells [53]. Likewise, treatment failure 
due to lack or loss of EBV antigen expression by neoplastic cell subpopulations may be avoided through redirecting EBV-specific CTLs to target other tumor antigens. It has been shown that EBV-specific CTLs expressing a chimeric antigen receptor (CAR) specific for CD30, a molecule highly and consistently expressed on malignant Hodgkin Reed-Sternberg cells, while retaining their original specificity, are also able to target $\mathrm{CD} 30+$ neoplastic cells, and mediate activity against EBV-/CD30+ tumors in a xenograft model [54].

\section{Conclusions}

The management of human cancer with radio-chemotherapy is still suboptimal, due to persistence of refractory/relapsing disease, and the increased toxicity observed with increased efficacy of therapeutic regimens. Targeted therapies may offer equal or increased efficacy, coupled with a considerable decrease in overall toxicity. Among these novel approaches, cell therapy offers a unique opportunity to restore antitumor immune surveillance, and it is therefore conceivable that application of this strategy will increase in the next few years [55]. In particular, for patients with relapsed or refractory NPC which express EBV antigens, autologous, polyclonal, EBV-specific CTL therapy is safe, feasible, with low complication rates and a significant clinical response.

\section{Acknowledgments}

This work was supported in part by grants from AIRC (Associazione Italiana Ricerca sul Cancro) to PC; Ministero della Salute, Progetti Ricerca Oncologica number RFPS-2006-4-341763 to PC; RFPS-2006-Regione Umbria to PC and MZ; Progetti Ricerca Finalizzata to PC and MZ; Fondazione IRCCS Policlinico S. Matteo, progetti Ricerca Corrente to MZ; AIFA (Agenzia Italiana del Farmaco).

\section{Conflict of Interest}

The authors have declared that no conflict of interest exists.

\section{References}

1. Rosenberg SA, Restifo NP, Yang JC, et al. Adoptive cell transfer: a clinical path to effective cancer immunotherapy. Nat Rev Cancer. 2008; 8: 299-308.

2. Blattman JN, Greenberg PD. Cancer immunotherapy: a treatment for the masses. Science. 2004; 305: 200-5.

3. Leen AM, Rooney CM, Foster AE. Improving T cell therapy for cancer. Annu Rev Immunol. 2007; 25: 243-65.

4. Rosenberg SA, Lotze MT, Muul LM, et al. Observations on the systemic administration of autologous lymphokine-activated killer cells and recombinant interleukin-2 to patients with metastatic cancer. N Engl J Med. 1985; 313: 1485-92.
5. Sangiolo D, Mesiano G, Carnevale-Schianca F, et al. Cytokine induced killer cells as adoptive immunotherapy strategy to augment graft versus tumor after hematopoietic cell transplantation. Expert Opin Biol Ther. 2009 Jul;9(7):831-40.

6. Rosenberg SA, Packard BS, Aebersold PM, et al. Use of tumor-infiltrating lymphocytes and interleukin-2 in the immunotherapy of patients with metastatic melanoma. A preliminary report. N Engl J Med. 1988; 319: 1676-80.

7. Dudley ME, Yang JC, Sherry R, et al. Adoptive cell therapy for patients with metastatic melanoma: evaluation of intensive myeloablative chemoradiation preparative regimens. J Clin Oncol. 2008; 26: 5233-9.

8. Shablak A, Hawkins RE, Rothwell DG, Elkord E. T cell-based immunotherapy of metastatic renal cell carcinoma: modest success and future perspective. Clin Cancer Res 2009;15:6503-10.

9. Heslop HE, Slobod KS, Pule MA, Hale GA, Rousseau A, Smith CA, et al. Long-term outcome of EBV-specific T-cell infusions to prevent or treat EBV-related lymphoproliferative disease in transplant recipients. Blood 2010; 115:925-935

10. Comoli P, Labirio M, Basso S, et al. Infusion of autologous Epstein-Barr virus (EBV)-specific cytotoxic T cells for prevention of EBV-related lymphoproliferative disorder in solid organ transplant recipients with evidence of active virus replication. Blood. 2002; 99: 2592-8.

11. Haque T, Wilkie GM, Jones MM, Higgins CD, Urquhart G, Wingate $\mathrm{P}$, et al. Allogeneic cytotoxic T-cell therapy for EBV-positive posttransplantation lymphoproliferative disease: results of a phase 2 multicenter clinical trial. Blood 2007; 110:1123-1131.

12. Spano JP, Busson $P$, Atlan D, et al. Nasopharyngeal carcinomas: an update. Eur J Cancer. 2003; 39: 2121-35.

13. Tao Q, Chan AT. Nasopharyngeal carcinoma: molecular pathogenesis and therapeutic developments. Expert Rev Mol Med. 2007; 9: 1-24.

14. Ayan I, Kaytan E, Ayan N. Childhood nasopharyngeal carcinoma: from biology to treatment. Lancet Oncol. 2003; 4: 13-21.

15. Williams H, Crawford DH. Epstein-Barr virus: the impact of scientific advances on clinical practice. Blood. 2006; 107: 862-9.

16. Roebuck, DJ. Skeletal complications in pediatric oncology patients. Radiographics. 1999; 19: 873-85.

17. Bass IS, Haller JO, Berdon WE, et al. Nasopharyngeal carcinoma: clinical and radiographic findings in children. Radiology. 1985; 156: 651-4.

18. Cvitkovic E, Bachouchi M, Boussen H, et al. Leukemoid reaction, bone marrow invasion, fever of unknown origin, and metastatic pattern in the natural history of advanced undifferentiated carcinoma of nasopharyngeal type: a review of 255 consecutive cases. J Clin Oncol. 1993; 11: 2434-42.

19. Perez CA, Devineni VR, Marcial-Vega V, et al. Carcinoma of the nasopharynx: factors affecting prognosis. Int J Radiat Oncol Biol Phys. 1992; 23: 271-80.

20. Ozyar E, Selek U, Laskar S, et al. Treatment results of 165 pediatric patients with non-metastatic nasopharyngeal carcinoma: a Rare Cancer Network study. Radiother Oncol. 2006; 81: 39-46.

21. Orbach D, Brisse H, Helfre S, et al. Radiation and chemotherapy combination for nasopharyngeal carcinoma in children: Radiotherapy dose adaptation after chemotherapy response to minimize late effects. Pediatr Blood Cancer. 2008; 50: 849-53.

22. Wolden SL, Zelefsky MJ, Hunt MA, et al. Failure of a 3D conformal boost to improve radiotherapy for nasopharyngeal carcinoma. Int J Radiat Oncol Biol Phys. 2001; 49: 1229-34.

23. Lee N, Xia P, Quivey JM, et al. Intensity-modulated radiotherapy in the treatment of nasopharyngeal carcinoma: an update of the UCSF experience. Int J Radiat Oncol Biol Phys. 2002; 53: $12-22$. 
24. Hsiung CY, Yorke ED, Chui CS, et al. Intensity-modulated radiotherapy versus conventional three-dimensional conformal radiotherapy for boost or salvage treatment of nasopharyngeal carcinoma. Int J Radiat Oncol Biol Phys. 2002; 53: 638-47.

25. Song $\mathrm{CH}, \mathrm{Wu} \mathrm{HG}$, Heo DS, et al. Treatment outcomes for radiotherapy alone are comparable with neoadjuvant chemotherapy followed by radiotherapy in early-stage nasopharyngeal carcinoma. Laryngoscope. 2008; 118: 663-70.

26. Al-Sarraf M, LeBlanc M, Giri PG, et al. Chemoradiotherapy versus radiotherapy in patients with advanced nasopharyngeal cancer: phase III randomized Intergroup study 0099. J Clin Oncol. 1998; 16: 1310-7.

27. Cheng SH, Tsai SY, Yen KL, et al. Concomitant radiotherapy and chemotherapy for early-stage nasopharyngeal carcinoma. J Clin Oncol. 2000; 18: 2040-5.

28. Langendijk JA, Leemans CR, Buter J, et al. The additional value of chemotherapy to radiotherapy in locally advanced nasopharyngeal carcinoma: a meta-analysis of the published literature. J Clin Oncol. 2004; 22: 4604-12.

29. Wei WJ, Sham JST. Nasopharyngeal carcinoma. Lancet 2005; 365: 2041-2054.

30. Chan AT, Hsu MM, Goh BC, et al. Multicenter, phase II study of cetuximab in combination with carboplatin in patients with recurrent or metastatic nasopharyngeal carcinoma. J Clin Oncol. 2005; 23:1-9.

31. Forastiere A, Koch W, Trotti A, et al. Head and neck cancer. N Engl J Med. 2001; 345: 1890-900.

32. Arango BA, Castrellon AB, Perez CA, et al. Nasopharyngeal carcinoma: alternative treatment options after disease progression. Expert Rev Anticancer Ther. 2010; 10: 377-86.

33. Comoli P, Zecca M, Maccario R. Immunotherapy against EBV-lymphoma in recipients of HSCT. Expert Rev Hematol. 2010;3(5):625-632.

34. Merlo A, Turrini R, Dolcetti R, Martorelli D, Muraro E, Comoli $\mathrm{P}$, Rosato A. The interplay between Epstein-Barr virus and the immune system: a rationale for adoptive cell therapy of EBV-related disorders. Haematologica. 2010; 95:1769-77.

35. Comoli P, Pedrazzoli P, Maccario R, et al. Cell therapy of stage IV nasopharyngeal carcinoma with autologous Epstein-Barr virus-targeted cytotoxic T lymphocytes. J Clin Oncol. 2005; 23: 8942-9.

36. Straathof KC, Bollard CM, Popat U, et al. Treatment of nasopharyngeal carcinoma with Epstein-Barr virus--specific $\mathrm{T}$ lymphocytes. Blood. 2005; 105: 1898-904.

37. Louis CU, Straathof K, Bollard CM, et al. Adoptive transfer of EBV-specific $\mathrm{T}$ cells results in sustained clinical responses in patients with locoregional nasopharyngeal carcinoma. J Immunother. 2010; 33: 983-90.

38. Louis CU, Straathof $\mathrm{K}$, Bollard CM, et al. Enhancing the in vivo expansion of adoptively transferred EBV-specific CTL with lymphodepleting CD45 monoclonal antibodies in NPC patients. Blood. 2009; 113: 2442-50.

39. Secondino S. et al. T-cell therapy for ebv-associated nasopharyngeal carcinoma: preparative lymphodepleting chemotherapy does not improve clinical results. Ann Oncol. 2011; in press.

40. Chua D, Huang J, Zheng B, et al. Adoptive transfer of autologous Epstein-Barr virus- specific cytotoxic T cells for nasopharyngeal carcinoma. Int J Cancer. 2001; 94:73-80

41. Comoli P, De Palma R, Siena S, et al. Adoptive transfer of allogeneic EBV-specific cytotoxic T cells with in vitro antitumor activity boosts LMP-2-specific immune response in a patient with EBV-related nasopharyngeal carcinoma. Ann Oncol. 2004; 15:113-117.

42. Rosenberg SA, Dudley ME. Adoptive cell therapy for the treatment of patients wit.h metastatic melanoma. Curr Opin Immunol. 2009; 21: 233-40.
43. Dudley ME, Wunderlich JR, Yang JC, et al. Adoptive cell transfer therapy following nonmyeloablative but lymphodepleting chemotherapy for the treatment of patients with refractory metastatic melanoma. J Clin Oncol. 2005;23:2346-2357.

44. Lee AW, et al. Retrospective analysis of patients with nasopharyngeal carcinoma treated during 1976-1985: survival after local recurrence. Int J Radiat Oncol Biol Phys 1993; 26: 773-82.

45. Hasbini A, et al.. Phase II trial combining mitomycin with 5-fluorouracil, epirubicin, and cisplatin in recurrent and metastatic undifferentiated carcinoma of nasopharyngeal type. Ann Oncol, 1999; 10:421-5.

46. Yee C, Thompson JA, Byrd D, et al. Adoptive T cell therapy using antigen-specific CD8+ $\mathrm{T}$ cell clones for the treatment of patients with metastatic melanoma: in vivo persistence, migration, and antitumor effect of transferred T cells. Proc Natl Acad Sci U S A. 2002; 99: 16168-73.

47. Gottschalk S, Ng CY, Perez M, et al. An Epstein-Barr virus deletion mutant associated with fatal lymphoproliferative disease unresponsive to therapy with virus-specific CTLs. Blood. 2001; 97: 835-43.

48. Gottschalk S, Edwards OL, Sili U, et al. Generating CTLs against the subdominant Epstein-Barr virus LMP1 antigen for the adoptive immunotherapy of EBV-associated malignancies. Blood. 2003; 101: 1905-12.

49. Gahn B, Siller-Lopez F, Pirooz AD, et al. Adenoviral gene transfer into dendritic cells efficiently amplifies the immune response to LMP2A antigen: a potential treatment strategy for Epstein-Barr virus--positive Hodgkin's lymphoma. Int J Cancer. 2001; 93: 706-13.

50. Ranieri E, Herr W, Gambotto A, et al. Dendritic cells transduced with an adenovirus vector encoding Epstein-Barr virus latent membrane protein 2B: a new modality for vaccination. J Virol. 1999; 73: 10416-25.

51. Bollard CM, Gottschalk S, Leen AM, et al. Complete responses of relapsed lymphoma following genetic modification of tumor-antigen presenting cells and T-lymphocyte transfer. Blood. 2007; 110: 2838-45.

52. Drake CG, Jaffee E, Pardoll DM. Mechanisms of immune evasion by tumors. Adv Immunol. 2006;90:51-81.

53. Bollard CM, Rossig C, Calonge MJ, et al. Adapting a transforming growth factor beta-related tumor protection strategy to enhance antitumor immunity. Blood. 2002;99:3179-3187.

54. Savoldo B, Rooney CM, Di Stasi A, et al. Epstein Barr virus-specific cytotoxic $\mathrm{T}$ lymphocytes expressing the anti-CD30 artificial chimeric T-cell receptor for immunotherapy of Hodgkin disease. Blood. 2007;110:2620-2630.

55. Comoli P. Cell-based therapies: careful safety assessment for minimization of risk. Cytotherapy. 2010;12:710-2. 